\title{
Cultivo de larvas de Ucides cordatus (LINNAEU, 1763) sob diferentes intensidades luminosas
}

\author{
[Rearing of Ucides cordatus (LINNAEU, 1763) larvae \\ under different light intensities] \\ K.F. Cottens $^{1}$, U.A.T. Silva ${ }^{2}$, R. Ventura ${ }^{3}$, F.M. Ramos ${ }^{4}$, A. Ostrensky ${ }^{5}$ \\ ${ }^{1}$ Instituto Chico Mendes de Conservação da Biodiversidade - ICMBio/MMA- Brasília, DF \\ ${ }^{2}$ Universidade Federal do Paraná -UFPR/Camar - Pontal do Sul, PR \\ ${ }^{3}$ Empresa de Pesquisas Agronômicas do Estado de Santa Catarina - Epagri - Florianópolis, SC \\ ${ }^{4}$ Aluno de pós-graduação - Universidade Federal do Pará - UFPA - Belém, PA \\ ${ }^{5}$ Universidade Federal do Paraná - Curitiba, PR
}

\begin{abstract}
RESUMO
O caranguejo-uçá, Ucides cordatus, é uma espécie típica dos manguezais brasileiros e tem grande importância econômica para as populações litorâneas tradicionais. O presente trabalho investigou a influência da intensidade luminosa sobre a sobrevivência e a taxa de desenvolvimento larval de $U$. cordatus. Três intensidades luminosas foram avaliadas: claro - 710 lux, penumbra -210 lux e escuro - 1 lux, em duas condições de cultivo, individual e coletivo. Houve diferenças significativas entre as taxas de sobrevivência das larvas zoea e as três intensidades luminosas avaliadas $(\mathrm{p}<0,05)$. As maiores taxas de ecdise para o estágio de megalopa foram obtidas no tratamento claro (42\% nos cultivos coletivos e $30 \%$ nos cultivos individuais). No tratamento escuro, a metamorfose para megalopa foi de apenas $16 \%$ nos cultivos coletivos e de $7 \%$ nos cultivos individuais. Estes resultados indicam que a manutenção das larvas em baixas intensidades luminosas afeta negativamente a sobrevivência larval de $U$. cordatus.
\end{abstract}

Palavras-chave: desenvolvimento larval, intensidade luminosa, larvicultura, sobrevivência, Ucides cordatus

\begin{abstract}
Ucides cordatus is an edible crab species typical of Brazilian mangroves, and traditionally represents an important economic resource for many coastal populations. The present study investigated the influence of light intensity on the survival and rate of larval development of $\mathrm{U}$. cordatus. Three different levels of luminosity were evaluated: 710 (Light), 210 Lux (Shaded) and 1 Lux (Dark), both in individual and collective cultivation conditions. Significant differences were found for survival of zoea larvae under the different light intensities $(P<0.05)$. The greatest survival rates as well as rates of ecdysis to the megalopa stage were obtained under Light conditions (42\% in collective cultures and $30 \%$ in individual cultures). In Dark conditions events of metamorphosis to megalopa stage was observed only in 16\% of collective cultures and $7 \%$ of individual cultures. The result indicates that low light intensities may negatively affect larval survivorship during U. cordatus larval cultivations.
\end{abstract}

Keywords: larval development, light intensity, larviculture, survival, ucides cordatus

\section{INTRODUÇÃO}

O caranguejo-uçá, Ucides cordatus (Linnaeus, 1763), habita os manguezais da costa atlântica das Américas (Melo, 1996). É um crustáceo de

Recebido em 21 de março de 2013

Aceito em 11 de fevereiro de 2014

E-mail: kelly.cottens@icmbio.gov.br grande importância econômica para pescadores artesanais e extrativistas (Glaser, 2003). Segundo dados oficiais, o caranguejo-uçá foi o terceiro crustáceo mais capturado em 2010, com o total de 8535 toneladas, ou $14,93 \%$ da produção nacional de crustáceos (Brasil, 2012). 
Assim como muitas espécies de pescados, os estoques do caranguejo-uçá já apresentam redução em diversas regiões do Brasil (Alves e Nishida, 2003). Esse fato pode estar relacionado a fatores como o extrativismo excessivo, a poluição, a destruição das áreas de manguezal e a doença do caranguejo letárgico (Boeger et al., 2005).

Uma das estratégias utilizadas para a recuperação das populações do caranguejo-uçá é o repovoamento. Esse processo consiste na soltura de larvas ou juvenis da espécie-alvo do repovoamento em seu ambiente natural. O Grupo Integrado de Aquicultura e Estudos Ambientais da Universidade Federal do Paraná (Gia/UFPR) realiza estudos para o desenvolvimento de tecnologias para o cultivo de larvas de $U$. cordatus destinadas ao repovoamento (Silva, 2007).

Esforços para a produção de grandes quantidades de larvas e juvenis de espécies nativas, com o objetivo de recuperar populações outrora abundantes, também já foram empreendidos em outros países, como nos Estados Unidos, onde o repovoamento foi realizado na baía de Chesapeake (Zmora et al., 2005), repovoada com juvenis do siri- azul, Callinectes sapidus, e em áreas no Pacífico Norte, que receberam juvenis de duas espécies de caranguejo-real ou "king crab", Paralithodes camtschaticus e P. brevipes (Kittaka et al., 2002). No Japão, o repovoamento de áreas de manguezal com juvenis do siri Scylla serrata vem sendo realizado desde 1979 (Davis, 2004).

As larvas zoea apresentam fototropismo positivo bem acentuado (Queiroga et al., 1997; Anger, 2001). No ambiente natural, elas realizam migrações verticais e tendem a se manter em regiões subsuperficiais, com intensidade luminosa moderada e abundância de alimento (Pittman e McAlpine, 2003; Queiroga e Blanton, 2005; Pineda et al., 2006).

No ambiente de cultivo, devido à profundidade reduzida e à relativa homogeneidade ambiental (McVey, 1986), as condições de iluminação são bastante distintas daquelas observadas no ambiente natural, o que pode refletir na sobrevivência e na qualidade das larvas cultivadas.
O presente trabalho tem como objetivo avaliar os efeitos de diferentes condições de iluminação durante o cultivo larval de $U$. cordatus sobre as taxas de sobrevivência e sobre o tempo necessário para o desenvolvimento até o estágio de megalopa, quando as larvas estão naturalmente aptas para a liberação nas áreas de manguezal.

\section{MATERIAL E MÉTODOS}

Os experimentos foram realizados nos meses de janeiro e fevereiro de 2008, no Laboratório de Pesquisa com Organismos Aquáticos LapoaGia/UFPR, localizado na cidade de Curitiba, Paraná.

Fêmeas de $U$. cordatus com ovos foram coletadas por pescadores em áreas de manguezal do Complexo Estuarino de Paranaguá (2525S, $48^{\circ} 230$ ). Ao chegarem ao laboratório, os animais foram lavados para retirada do sedimento de manguezal e passaram por desinfecção, utilizando-se solução de iodo $0,02 \%$. A seguir, foram acondicionados em tanques de eclosão com capacidade para 1000L, preenchidos com aproximadamente $250 \mathrm{~L}$ de água do mar esterilizada e filtrada, com salinidade ajustada a 30PSU, temperatura de $25 \pm 1^{\circ} \mathrm{C}$, fotoperíodo de $14 \mathrm{~L}: 10 \mathrm{E}$ e aeração constante. Para a realização deste experimento, foram montados três tanques de eclosão e cada um recebeu de 15 a 20 fêmeas.

Após a eclosão, as larvas zoea foram concentradas, utilizando-se uma fonte luminosa, e coletadas por sifonamento. As larvas eclodidas no mesmo dia em todos os tanques de eclosão foram agrupadas em tanques de fibra de vidro com capacidade de 100L. A seguir, as larvas passaram por um período de 24 horas sem alimentação, quando aquelas larvas que naturalmente eram inviáveis acabavam morrendo e eram descartadas. Após esse período, as larvas mais ativas foram coletadas ao acaso, utilizandose uma pipeta de Pasteur com ponta alargada, e utilizadas para povoar os experimentos.

Dois experimentos foram realizados, ambos avaliando três níveis de intensidade luminosa: claro -710 lux, penumbra -210 lux e escuro -1 lux. O primeiro experimento foi realizado em frascos plásticos, com volume individual de $200 \mathrm{~mL}$, povoados com 15 larvas, sendo cada 
tratamento testado com 15 repetições, em um total de 45 unidades experimentais.

O segundo experimento foi realizado em cultivos individuais, utilizando-se frascos plásticos com volume de $20 \mathrm{~mL}$, sendo cada tratamento testado com 30 repetições, em um total de 90 unidades experimentais.

A fonte luminosa empregada nos experimentos foi constituída por um conjunto de lente e colimador com 10 Leds ("Light Emitting Diode") de luz branca. A luz emitida é compatível com a luz solar, e a intensidade luminosa foi regulada com o uso de um dimer. O tratamento escuro foi mantido em um ambiente sem qualquer fonte luminosa.

Os experimentos foram realizados em sala climatizada, com temperatura de $25 \pm 1^{\circ} \mathrm{C}$, fotoperíodo 14L:10E e salinidade 30PSU . Durante os experimentos, as larvas foram alimentadas com a microalga Chaetoceros muelleri (400.000 células. $\mathrm{mL}^{-1}$ ) e os rotíferos Brachionus plicatilis $\left(20\right.$ indivíduos. $\left.\mathrm{mL}^{-1}\right)$, seguindo as recomendações de Becker (2008).

A cada $24 \mathrm{~h}$ era realizada a manutenção dos experimentos, quando as larvas eram retiradas das unidades experimentais e transferidas para frascos posicionados sobre uma bandeja de triagem. Nessa fase, as larvas mortas eram quantificadas e excluídas do experimento. As unidades experimentais passavam por higienização e depois recebiam uma nova solução de cultivo, composta por água e organismos-alimento. A seguir, as larvas eram devolvidas às suas respectivas unidades experimentais.

Para o manejo das larvas do tratamento escuro, foi utilizada uma luminária de mesa com lâmpada fluorescente branca, e cada unidade experimental foi manejada individualmente, o mais rápido possível, diminuindo a exposição das larvas à luz durante esse processo.

Os experimentos foram encerrados quando a última larva zoea atingiu a fase de megalopa, ou quando não havia mais larvas vivas em nenhuma unidade experimental.

Os dados dos experimentos de cultivos coletivos e individuais foram tabulados e submetidos ao teste de normalidade de Shapiro-Wilk e, posteriormente, aos testes Kruskal-Wallis, Dunn e Kendall Tau, utilizando-se o software Statistica 6.0 (STATSOFT, 2004).

\section{RESULTADOS E DISCUSSÃO}

Os experimentos tiveram duração máxima de 36 dias nos cultivos coletivos e 41 dias nos cultivos individuais (Tab. 1). A taxa de sobrevivência final das larvas de $U$. cordatus foi dada pela ocorrência de metamorfose para a fase de megalopa.

Tabela 1. Ucides cordatus larvicultura em sistemas coletivo e individual, em três diferentes intensidades luminosas: claro - 710 lux, escuro - 1 lux e penumbra - 210 lux. As letras $(a, b, c)$ indicam os grupos homogêneos identificados por meio do teste de Kendall tau

\begin{tabular}{lccccc}
\hline & \multicolumn{5}{c}{ Intensidade luminosa } \\
\hline Cultivo coletivo & Unidade & Claro & Escuro & Penumbra & Total \\
\hline Duração do cultivo & dias & 36 & 32 & 36 & 36 \\
Quantidade inicial de zoeas & larvas & 225 & 225 & 225 & 675 \\
Primeira megalopa & dias & 25 & 24 & 24 & - \\
Última megalopa & dias & 37 & 33 & 37 & 13 \\
Total de megalopas & larvas & $95 \mathrm{a}$ & $35 \mathrm{~b}$ & $87 \mathrm{a}, \mathrm{b}$ & 217 \\
Sobrevivência final & $\%$ & $42 \mathrm{a}$ & $16 \mathrm{~b}$ & $39 \mathrm{a}, \mathrm{b}$ & 32 \\
Densidade de cultivo & larvas.L-1 & 75 & 75 & 75 & 75 \\
Cultivo individual & & & & & \\
Duração do cultivo & dias & 41 & 41 & 41 & 41 \\
Quantidade inicial de zoeas & larvas & 30 & 30 & 30 & 90 \\
Primeira megalopa & dias & 28 & 28 & 27 & - \\
Última megalopa & dias & 41 & 41 & 41 & 14 \\
Total de megalopas & larvas & $9 \mathrm{a}$ & $2 \mathrm{~b}$ & $7 \mathrm{~b}$ & 18 \\
Sobrevivência final & $\%$ & $30 \mathrm{a}$ & $7 \mathrm{~b}$ & $22 \mathrm{~b}$ & 20 \\
\hline
\end{tabular}


A sobrevivência dos cultivos coletivos foi significativamente maior $(p=0,0070)$ nos tratamentos claro (710 lux) e penumbra (210 lux) que no tratamento escuro (1 lux) (Figura 1-A). Nos cultivos individuais, a taxa de sobrevivência foi maior $(\mathrm{P}<0,001)$ no tratamento claro $(710$ lux), e os tratamentos escuro (1 lux) e penumbra (210 lux) apresentaram taxas de sobrevivência estatisticamente semelhantes (Figura 1-B).
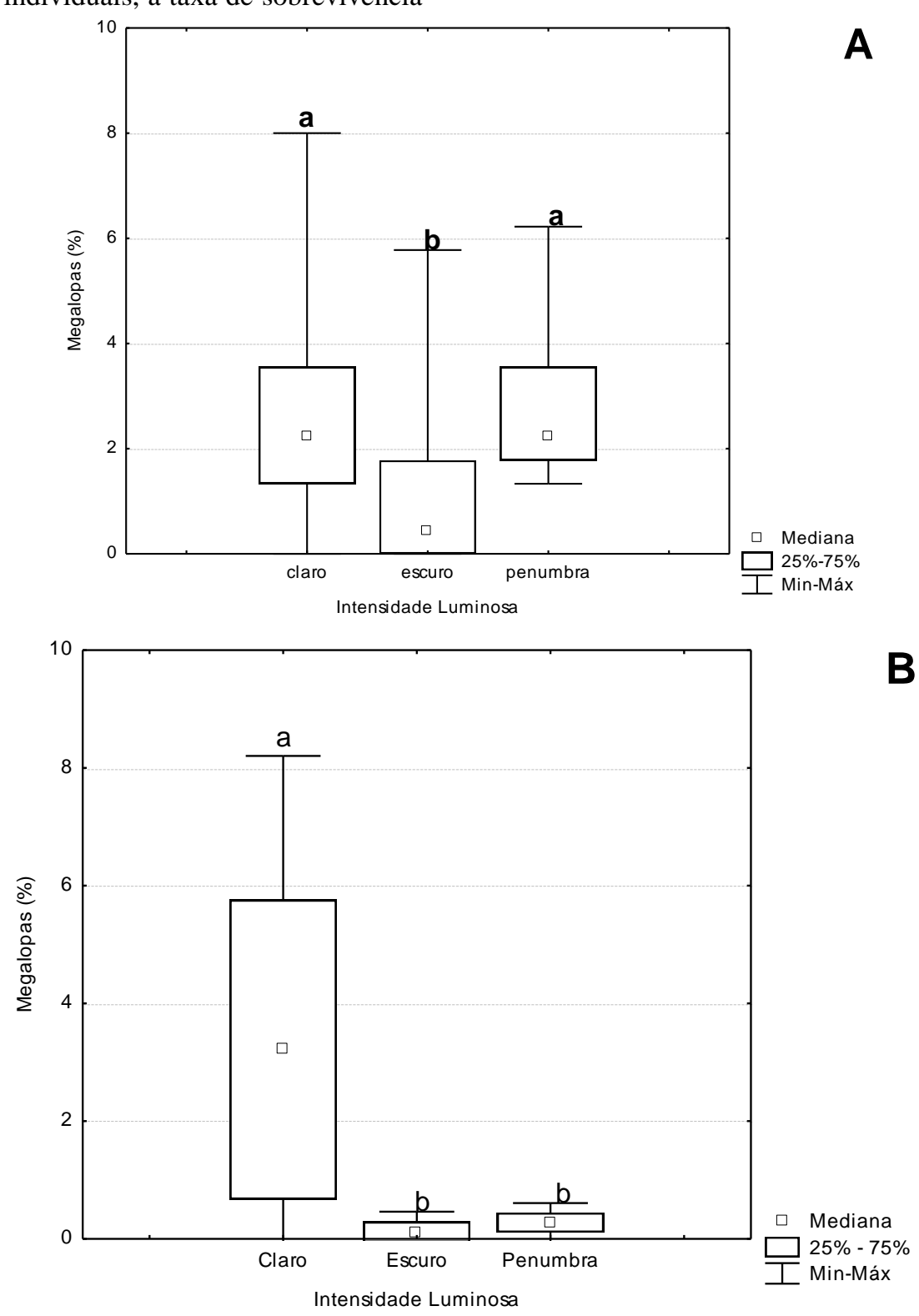

Figura 1. Ucides cordatus, sobrevivência das larvas zoea cultivadas em três diferentes condições de iluminação: claro - 710 lux, escuro - 1 lux e penumbra - 210 lux. A - cultivos coletivos; B - cultivos individuais. Letras iguais identificam tratamentos que não apresentaram diferenças significativas ao nível de 5\%, conforme teste de Dunn a posteriori.

Nos cultivos coletivos, a primeira megalopa foi observada no $24^{\circ}$ dia, no tratamento penumbra. As últimas megalopas foram coletadas no $32^{\circ}$ dia de cultivo no tratamento escuro e no $36^{\circ}$ dia nos demais tratamentos (Tab. 1). 
No cultivo individual, as primeiras megalopas ocorreram no $27^{\circ}$ dia no tratamento penumbra e no $28^{\circ}$ dia nos demais tratamentos. As últimas larvas megalopas foram coletadas no $41^{\circ}$ dia de cultivo em todos os tratamentos (Tab. 1).

A intensidade luminosa pode influenciar a taxa de crescimento e a duração da fase larval de crustáceos (Gardner e Maguire, 1998; Wang et al., 2004; Bermudes et al., 2008). Em laboratório, as larviculturas de crustáceos são realizadas em condições que costumam variar de 70 lux (Kittaka et al., 2002) até 1000 lux (Calado et al., 2003), conforme Tabela 2.

Tabela 2. Parâmetros utilizados no cultivo das larvas de diferentes espécies de crustáceos marinhos, incluindo U. cordatus

\begin{tabular}{|c|c|c|c|c|c|c|c|c|c|c|}
\hline 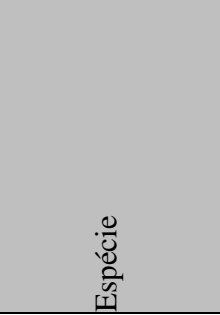 & 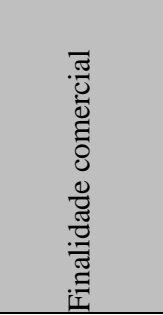 & 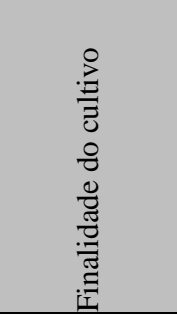 & 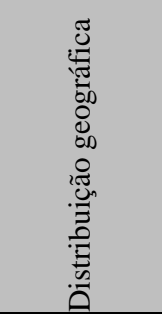 & 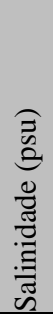 & 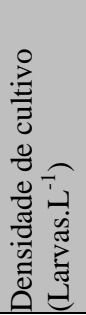 & 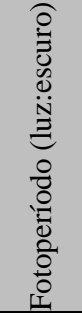 & 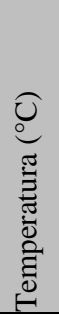 & 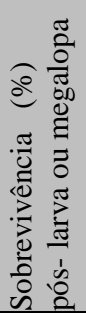 & 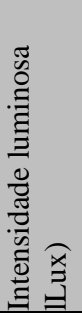 & 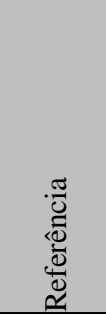 \\
\hline $\begin{array}{l}\text { Cryptodromiopsis } \\
\text { antillensis }\end{array}$ & Ornamental & $\begin{array}{l}\text { Comercial - } \\
\text { aquarismo }\end{array}$ & $\begin{array}{l}\text { Tropical - } \\
\text { recifes de } \\
\quad \text { coral }\end{array}$ & 35 & 18 & $14: 10$ & 22 & 20,6 & 1000 & $\begin{array}{l}\text { Calado } \\
\text { et al. } \\
(2003)\end{array}$ \\
\hline $\begin{array}{c}\text { Paralithodes } \\
\text { camtschaticus; } P . \\
\text { brevipes }\end{array}$ & Alimentação & $\begin{array}{l}\text { Acadêmica - } \\
\text { repovoamento }\end{array}$ & $\begin{array}{l}\text { Pacífico } \\
\text { Norte }\end{array}$ & 35 & $\begin{array}{c}10 \\
\mathrm{a} \\
50\end{array}$ & $12: 12$ & $\begin{array}{c}6 \mathrm{a} \\
9\end{array}$ & $\begin{array}{c}35 \mathrm{a} \\
53\end{array}$ & 70 & $\begin{array}{l}\text { Kittaka } \\
\text { et al. } \\
(2002)\end{array}$ \\
\hline
\end{tabular}

As diferenças observadas entre os experimentos realizados com cultivos coletivos e individuais, por sua vez, já foram observadas em outras espécies, como na larvicultura do caranguejoaranha, Maja brachydactyla, que apresenta maiores taxas de crescimento quando as larvas são cultivadas em densidades entre 50 e 100 larvas. $\mathrm{L}^{-1}$ em detrimento de densidades menores (Andrés et al., 2007). Para o cultivo de $U$. cordatus, recomendam-se densidades de 50 larvas. $\mathrm{L}^{-1}$ (Cottens et al., 2010).

No cultivo das larvas de $U$. cordatus, a sobrevivência foi maior nos cultivos realizados em intensidade luminosa elevada, tratamento claro (710 lux), o que demonstra que, para essa espécie, a alta intensidade luminosa pode contribuir para o aumento das taxas de sobrevivência.

Os efeitos da intensidade luminosa sobre a sobrevivência e o crescimento de crustáceos foram mais investigados no cultivo de camarões
(Wang et al., 2004). No cultivo de juvenis do camarão Penaeus semisulcatus, há um incremento nas taxas de sobrevivência em razão do aumento da intensidade luminosa (Al-Ablani e Farmer, 1986). Na larvicultura do camarão Litopenaeus vannamei, a taxa de crescimento larval é diretamente afetada pelo aumento da intensidade luminosa (You et al., 2006).

Por outro lado, a sobrevivência das larvas do caranguejo gigante australiano, Pseudocarcinus gigas (Gardner e Maguire, 1998), e da lagosta Jasus edwardsii (Bermudes et al., 2008) é pouco influenciada pela intensidade luminosa.

Essas observações são relacionadas a fatores ecológicos, pois a presença de outras larvas no sistema de cultivo estimula os comportamentos de competição, busca e captura de alimento, cria distúrbios em microescala que geram respostas sensoriais complexas, não sendo observadas quando as larvas são cultivadas individualmente (Browman, 2005). 
A capacidade de sobrevivência das larvas de crustáceos, mesmo em condições de baixíssima intensidade luminosa, pode estar relacionada com o mecanismo de captura de alimentos, especialmente quando a alimentação é feita por meio de um processo de filtração, como observado nas larvas de $U$. cordatus.

Assim como descrito por Anger (2001), a presença do estímulo luminoso torna as larvas de crustáceos mais ativas, o que promove um aumento em sua movimentação e, consequentemente, nas chances de encontrar partículas alimentares (Browman, 2005). Por outro lado, as larvas também são capazes de capturar alimentos em baixíssimos níveis de iluminação (Browman, 2005; Bermudes et al. 2008), quando apresentam um desenvolvimento mais lento, possivelmente relacionado aos baixos níveis de atividade, conforme verificado neste experimento. Durante o manejo das unidades amostrais, verificou-se que, para o tratamento escuro, as larvas de zoea cultivadas permaneciam no fundo das unidades amostrais na maior parte do tempo.

Essa observação também pode estar relacionada aos movimentos naturais de migração vertical realizados por larvas planctônicas, que, em situações de baixa intensidade luminosa, tendem a afundar passivamente na coluna d'água, buscando regiões mais profundas (Sulkin, 1984; Queiroga et al., 1997; Queiroga e Blanton, 2005; Pineda et al., 2006). Diferentemente do que ocorre nos sistemas artificiais de cultivo, em que as larvas permanecem no fundo dos tanques até que um novo estímulo promova sua natação e o deslocamento em direção aos estratos superiores da coluna d'água, nos cultivos realizados em laboratórios, o fundo do tanque tende a concentrar uma grande quantidade de matéria orgânica em decomposição e de microrganismos saprofíticos, o que afeta negativamente a sobrevivência e o desenvolvimento larval.

\section{CONCLUSÃO}

A larvicultura de $U$. cordatus apresenta melhores taxas de sobrevivência em condições de intensidade luminosa iguais ou superiores a 210 lux. Devem-se, portanto, evitar valores abaixo deste.

\section{AGRADECIMENTOS}

Ao Conselho Nacional de Pesquisa Científica $\mathrm{CNPq}$, pelo apoio financeiro recebido na forma de bolsa de estudos, ofertada pelo Programa de Pós-Graduação em Ciências Veterinárias da Universidade Federal do Paraná.

\section{REFERÊNCIAS}

Al-ABLANI, S.A.; FARMER, A.D.D. The effect of different levels of illuminance on the survival and growth of the shrimp Pennaeus semisulcatus. Kwuait Bul. of Marine Sci., p.165-172, 1986.

ALVES, R.N.; NISHIDA, A.K. Aspectos socioeconômicos e percepção ambiental dos catadores de caranguejo-uçá Ucides cordatus cordatus (L. 1763) (Decapoda, Brachyura) do estuário do rio Mamanguape, nordeste do Brasil. Interciência, v.28, p.36-43, 2003 .

ANDRÉS, M.; ESTÉVEZ, A.; ROTLANT, G. Growth, survival and biochemical composition of spider crab Maja brachydactyla (Balss, 1922) (Decapoda: Majidae) larvae reared under different stocking densities, prey: larva ratios and diets. Aquaculture, v. 273, p.35-40, 2007.

ANGER, K. The biology of decapod crustacean larvae. Crustacean Issues, v.14, p.420, 2001.

BECKER, A.G. Utilização de organismos-alimento na larvicultura do Caranguejo-uçá, Ucides cordatus (Linnaeus, 1763) (CRUSTACEA, BRACHYURA, OCYPODIDAE). 2008. 50f. Dissertação (Mestrado em Ciências Veterinárias) - Universidade Federal do Paraná, Curitiba.

BERMUDES, M.; RITAR, A.J.; CARTER, C.G. The ontogeny of physiological response to light intensity in early stage spiny lobster (Jasus edwardsii) larvae. Comp. Biochem. Physiol. A Mol. Integr. Physiol., v.150, p.40-45, 2008.

BOEGER, W.A.; PIE, M.R.; OSTRENSKY, A.; PATELLA, L. Lethargic crab disease: multidisciplinary evidence supports a mycotic etiology. Memórias do Instituto Oswaldo Cruz, v.100, p.161-167, 2005.

BROWMAN, H.I. Applications of sensory biology in marine ecology and aquaculture. Marine Ecol. Prog. Series, v.28, p.263-307, 2005.

CALADO, R.; NARCISO, L.; MORAIS, S. et al. A rearing system for the culture of ornamental decapod crustacean larvae. Aquaculture, v.218, p.329-339, 2003. 
COTTENS, K.F.; SILVA, U.A.; VENTURA, R. et al. Sobrevivência e desenvolvimento das larvas de Ucides cordatus (Linnaeus 1763) produzidas em laboratório sob diferentes densidades. In: Cyrino, J.E.P.; Furuya, W.M.; Ribeiro, R.P.; Scorvo, J.D. Tópicos Especiais Em Biologia Aquática e Aquicultura III, 2010. Sociedade Brasileira de Aquicultura e Biologia Aquática, Jaboticabal, 2010. cap. 31, p.321-328.

DAVIS, J.A. Development of hatchery techniques for the mud crab Scylla serrata (Forskál) in South Africa. Bélgica, 2004. 165 f. Tese (Doutorado (Ph. D.) em biologia aplicada) - Universiteit Gent, Faculdade de Biologia aplicada.

GARDNER, C.; MAGUIRE, G.B. Effect of fotoperiod and light intensity on survival, development and cannibalism of larvae of the giant crab Pseudocarcinus gigas (Lamarck). Aquaculture, v.165, p.51-53, 1998.

GLASER, M. Interrelations between mangrove ecosystem, local economy and social sustainability in Caeté Estuary, North Brazil. Wetlands Ecology Managemente, v.11, p.265-272, 2003.

KITTAKA, J.; STEVENS, B.G.; TESHIMA, S.; ISHIKAWA, M. Larval culture of the king crabs Paralithodes camtschaticus and P. brevipes. In: Crabs in cold water regions: biology, management, and economics, 2002. Alaska. p. 189-209.

McVEY, J. Handbook of Mariculture. Boca Raton, Florida, USA: CRC Press Inc. 1986. 154p.

MELO, G.A.S. Manual de identificação dos Brachyura (caranguejos e siris) do litoral brasileiro. São Paulo: PLÊIADE/FAPESP, 1996. 604p.

BRASIL. MINISTÉRIO DA PESCA E AQUICULTURA - MPA. Boletim Estatístico da Pesca e Aquicultura, Brasil 2010. Governo Federal, Brasilia, p.129, 2012.

PINEDA, J.; STARCZAK, V.; STUECLE, T.A. Timing of successful settlement: demonstration of a recruitment window in the barnacle Semibalanus balanoides. Marine Ecol. Progress Series, v.320, p.233-237, 2006.
PITTMAN, S.J.; McALPINE, C.A. Movements of marine fish and decapod crustaceans: process, theory and application. Adv. Marine Biol., v.44, p.205-280, 2003.

QUEIROGA, H.; BLANTON, J. Interactions between behavior and physical forcing in the control of horizontal transport of decapod crustacean larvae. $A d v$. Marine Biol., v.47, p.107-214, 2005.

QUEIROGA, H.; COSTLOW, J.D.; MOREIRA, M.H. Vertical migration of the crab Carcinus maenas in an estuary: implications for tidal stream transport. Marine Ecol. Progress Series, v.149, p.121-132, 1997.

SILVA, U.A. Recuperação populacional do caranguejo-uçá, Ucides cordatus (LINNAEUS, 1763), através da liberação de formas imaturas em áreas antrópicamente pressionadas. 2007. 174f. Tese (Doutorado em Zoologia), Universidade Federal do Paraná, Curitiba.

STATSOFT, Inc. STATISTICA (data analysis software system), version 6. Disponível em: www.statsoft.com. Acessado em: 2004.

SULKIN, S.D. Behavioral basis of depth regulation in the larvae of Brachyuran crabs. Marine Ecol. Prog. Series, v.15, p.181-205, 1984.

WANG, F.; DONG, S.; DONG, S. et al. The effect of light intensity on the growth of Chinese shrimp Fenneropenaeus chinensis. Aquaculture, v.234, p.475483, 2004.

YOU, K.; YANG, H.; LIU, Y. et al. Effects of different light sources and illumination methods on growth and body color of shrimp Litopenaeus vannamei. Aquaculture, v.252, p.557-565, 2006.

ZMORA, O.; FINDIESEN, A.; STUBBLEFIELD, J.; FRENKEL, V.; ZOHAR, Y. Large-scale juvenile production of the blue crab Callinectes sapidus. Aquaculture, v.244, p. 129-139. 2005. 